\title{
The Polar Equation of the Tangent and Normal to a Conic
}

By Robert J. T. Bell.

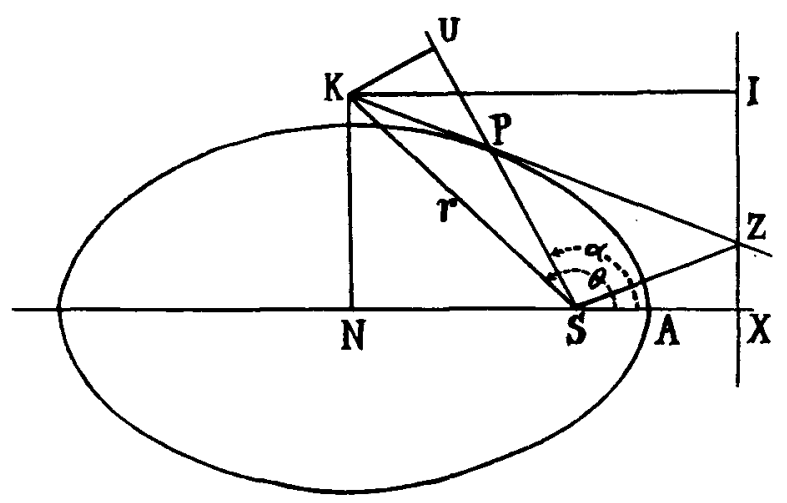

The property of a conic: " if the projections of $K$, any point on a tangent, on the directrix and the focal radius of the point of contact be $I$ and $U$ respectively, $S U=e . K I$," is known as Adams's Property. The statement of the above property in terms of the polar coordinates of $K$ is the equation of the tangent. Hence, if the eccentric angle of the point of contact is a

$$
\begin{aligned}
r \cos (\theta-a) & =e \cdot K I=e \cdot N X=e(N S+S X) \\
& =-e r \cos \theta+b,
\end{aligned}
$$

or $\frac{l}{r}=\cos (\theta-a)+e \cos \theta$ is the equation of the tangent.

It was thus suggested that there would be a corresponding property of the normal from which its equation could be derived. The property is as follows:

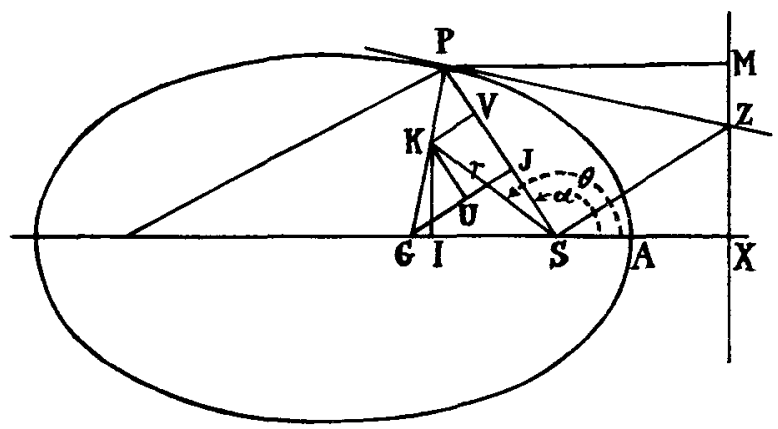

If $K$ is any point on the normal at $P$ to a conic and $I$ and $U$ are the 
$\mathrm{vi}$

projections of $K$ on the axis and on the perpendicular through $G$ to $S P$ respectively, $G U=e . K I$.

From the similar triangles $K G I, P Z M, \frac{K I}{K G}=\frac{P M}{P Z}$,

$$
K G U, P S Z, \frac{K G}{G U}=\frac{P Z}{S P}
$$

$$
\text { Therefore } \quad \frac{K I}{G U}=\frac{P M}{S P}=\frac{1}{e}, \quad \text { or } G U=e . K I \text {. }
$$

If $K$ is $(r, \theta)$ and if $K V$ is perpendicular to $S P$,

$$
K I=r \sin \theta, \quad K V=r \sin (\theta-a)
$$

and from the equation of the conic $S P=\frac{l}{1+e \cos \alpha}$.

Again $\quad G J=G S \cdot \sin \alpha=e \cdot S P \sin \alpha$.

Therefore er $\sin \theta+r \sin (\theta-\alpha)=\frac{e l \sin \alpha}{1+e \cos \alpha}$

or

$$
\frac{e \sin \alpha}{1+e \cos \alpha} \cdot \frac{l}{r}=\sin (\theta-\alpha)+e \sin \theta
$$

is the equation of the normal.

\section{A Problem in Confocals}

\section{By Robert J. T. Bell.}

In the standard text books on Analytical Geometry the following problem occurs in the exercises on confocals :

The product of the four normals from a point $P$ to the ellipse $x^{2} / a^{2}+y^{2} / b^{2}=1$ is equal to $\frac{\lambda_{1} \lambda_{2}\left(\lambda_{1}-\lambda_{2}\right)}{a^{2}-b^{2}}$ where $\lambda_{1}$ and $\lambda_{2}$ are the parameters of the confocals through $P$.

In the volume of solutions to the examples in C. Smith's "Conic Sections" two methods of solving the problem are given, the second of which, it is pointed out, is due to A. R. Forsyth. The following method is on much the same lines as Forsyth's, but seems to be more direct. 